\title{
OPERATOR POSITIVITY AND ANALYTIC MODELS OF COMMUTING TUPLES OF OPERATORS
}

\author{
MONOJIT BHATTACHARJEE AND JAYDEB SARKAR
}

\begin{abstract}
We study analytic models of operators of class $C_{.0}$ with natural positivity assumptions. In particular, we prove that for an $m$-hypercontraction $T \in C_{.0}$ on a Hilbert space $\mathcal{H}$, there exists two Hilbert spaces $\mathcal{E}$ and $\mathcal{E}_{*}$ and a partially isometric multiplier $\theta \in \mathcal{M}\left(H^{2}(\mathcal{E}), A_{m}^{2}\left(\mathcal{E}_{*}\right)\right)$ such that

$$
\mathcal{H} \cong \mathcal{Q}_{\theta}=A_{m}^{2}\left(\mathcal{E}_{*}\right) \ominus \theta H^{2}(\mathcal{E}), \quad \text { and }\left.\quad T \cong P_{\mathcal{Q}_{\theta}} M_{z}\right|_{\mathcal{Q}_{\theta}},
$$

where $A_{m}^{2}\left(\mathcal{E}_{*}\right)$ is the $\mathcal{E}_{*}$-valued weighted Bergman space and $H^{2}(\mathcal{E})$ is the $\mathcal{E}$-valued Hardy space over the unit disc $\mathbb{D}$. We then proceed to study and develop analytic models for doubly commuting $n$-tuples of operators and investigate their applications to joint shift co-invariant subspaces of reproducing kernel Hilbert spaces over polydisc. In particular, we completely analyze doubly commuting quotient modules of a large class of reproducing kernel Hilbert modules, in the sense of Arazy and Englis, over the unit polydisc $\mathbb{D}^{n}$.
\end{abstract}

\section{Notation}

$\mathbb{N} \quad$ Set of all natural numbers including 0 .

$n \quad$ Natural number $n \geq 2$.

$\mathbb{N}^{n} \quad\left\{\boldsymbol{k}=\left(k_{1}, \ldots, k_{n}\right): k_{i} \in \mathbb{N}, i=1, \ldots, n\right\}$.

$\boldsymbol{z} \quad\left(z_{1}, \ldots, z_{n}\right) \in \mathbb{C}^{n}$.

$z^{k} \quad z_{1}^{k_{1}} \cdots z_{n}^{k_{n}}$.

$\boldsymbol{T} \quad n$-tuple of commuting operators $\left(T_{1}, \ldots, T_{n}\right)$.

$\boldsymbol{T}^{k} \quad T_{1}^{k_{1}} \cdots T_{n}^{k_{n}}$.

$\mathbb{D}^{n} \quad$ Open unit polydisc $\left\{\boldsymbol{z}:\left|z_{i}\right|<1\right\}$.

For a closed subspace $\mathcal{S}$ of a Hilbert space $\mathcal{H}$, we denote by $P_{\mathcal{S}}$ the orthogonal projection of $\mathcal{H}$ onto $\mathcal{S}$. We shall denote the space of all bounded linear operators on $\mathcal{H}$ by $\mathcal{B}(\mathcal{H})$.

\section{INTRODUCTION}

The Sz.-Nagy and Foias analytic model theory for contractions on Hilbert spaces is a powerful tool for studying operators on Hilbert spaces and holomorphic function spaces on the open unit disc $\mathbb{D}$ in $\mathbb{C}$. It says that if $T$ is a contraction (that is, $I-T T^{*} \geq 0$ ) on a Hilbert space and in $C_{\text {. } 0}$ class (that is, $T^{* l} \rightarrow 0$ as $l \rightarrow \infty$ in strong operator topology) then $T^{*}$ is unitarily equivalent to the restriction of the backward shift $M_{z}^{*}$ on a vector-valued Hardy

2000 Mathematics Subject Classification. 47A13, 47A15, 47A20, 47A45, 47A80, 47B32, 47B38.

Key words and phrases. Weighted Bergman spaces, hypercontractions, multipliers, reproducing kernel Hilbert spaces, invariant subspaces. 
space to a $M_{z}^{*}$-invariant subspace. More precisely, there exists a coefficient Hilbert space $\mathcal{E}_{*}$ and a $M_{z}^{*}$-invariant closed subspace $\mathcal{Q}$ of $\mathcal{E}_{*}$-valued Hardy space $H^{2}\left(\mathcal{E}_{*}\right)$ such that

$$
\left.T \cong P_{\mathcal{Q}} M_{z}\right|_{\mathcal{Q}} .
$$

Moreover, there exists a Hilbert space $\mathcal{E}$ and a $\mathcal{B}\left(\mathcal{E}, \mathcal{E}_{*}\right)$-valued inner multiplier $\theta_{T} \in H_{\mathcal{B}\left(\mathcal{E}, \mathcal{E}_{*}\right)}^{\infty}(\mathbb{D})$, also known as the characteristic function of $T$ (see [13]), such that

$$
\mathcal{Q}=H^{2}\left(\mathcal{E}_{*}\right) / \theta_{T} H^{2}(\mathcal{E}) .
$$

In [1], Agler introduced and studied the theory hypercontraction operators from operator positivity point of view. He showed that the vector-valued Hardy space in the dilation space of a contraction can be replaced by a vector-valued weighted Bergman space if the contractivity assumption on the operator is replaced by a weighted Bergman type positivity. Later, Muller and Vasilescu [12], Curto and Vasilescu [11], Ambrozie and Timotin [3, 4], Arazy, Englis and Muller [2] and Arazy and Englis [5] extended these ideas to a more general class of operators. This viewpoint has proved to be extremely fruitful in studying commuting tuples of operators.

The purpose of this paper is to explore how one might do analytic model theory for a general class of operators and commuting tuples of operators. In particular, we associate a partially isometric multiplier with every operator satisfying weighted Bergman-type positivity condition (see Theorem 2.4). Another basic result in this direction is the following analytic model: Let $\boldsymbol{T}=\left(T_{1}, \ldots, T_{n}\right)$ be a doubly commuting tuple of pure operators on a Hilbert space $\mathcal{H}$ (that is, $T_{i} \in C_{.0}, T_{i} T_{j}=T_{j} T_{i}$ and $T_{p} T_{q}^{*}=T_{q}^{*} T_{p}$ for all $i, j=1, \ldots, n$, and $1 \leq p<q \leq n$ ). Then $\left(T_{1}^{*}, \ldots, T_{n}^{*}\right)$ is joint unitarily equivalent to the restriction of $\left(M_{z_{1}}^{*}, \ldots, M_{z_{n}}^{*}\right)$ to a joint invariant subspace of a vector-valued weighted Bergman space if and only if $T$ satisfies (joint) weighted Bergman type positivity. Moreover, in this case, the orthocomplement of the coinvariant subspace of the weighted Bergman space is of "Beurling-lax-Halmos" type (see Theorems 4.5).

Although our method works for more general cases (see Section 7), for simplicity we will restrict our discussion to hypercontractions (see Section 2).

Here is a brief description of the paper. In Section 2 we set up notations, recall some basic notions from the theory of hypercontractions and construct an analytic structure on the model space. Our main tool here is the Agler dilation theorem for hypercontractions [1] combined with a Beurling-Lax-Halmos type representations of shift invariant subspaces of analytic reproducing kernel Hilbert spaces ([8], [15]). In Section 3 we discuss a dilation theory for a class of doubly commuting operator tuples satisfying weighted Bergman type positivity condition. In Section 4 we formulate a version of Sz.-Nagy and Foias analytic model for doubly commuting tuple of hypercontractions. In Section 5, we analyze doubly commuting quotient modules of scalar valued weighted Bergman space. Finally in section 6 we study $K$-contractive tuples of operators in the spirit of Arazy and Englis [5].

\section{Functional Models for Hypercontractions}

The main purpose of this section is to study and to develop an analytic functional model for the class of hypercontractions on Hilbert spaces. 
We first recall the definition of weighted Bergman spaces and review the construction of the dilation maps for hypercontractions. We refer the reader to Agler's paper [1] for more details.

The weighted Bergman kernel on the open unit disc $\mathbb{D}$ with weight $\alpha>0$ is, by definition, the kernel function:

$$
B_{\alpha}(z, w)=(1-z \bar{w})^{-\alpha} \quad(z, w \in \mathbb{D}) .
$$

For each $\alpha>0$, we let $A_{\alpha}^{2}$, denote the weighted Bergman space corresponding to the kernel $B_{\alpha}$. For any Hilbert space $\mathcal{E}$, the $\mathcal{E}$-valued weighted Bergman space $A_{\alpha}^{2}(\mathcal{E})$ with reproducing kernel $(z, w) \in \mathbb{D} \times \mathbb{D} \mapsto B_{\alpha}(z, w) I_{\mathcal{E}}$ can canonically be identified with the Hilbert space tensor product $A_{\alpha}^{2} \otimes \mathcal{E}$. In order to simplify notation, we often identify $A_{\alpha}^{2} \otimes \mathcal{E}$ with $A_{\alpha}^{2}(\mathcal{E})$. It also follows that $\left\{B_{\alpha}(\cdot, w) \eta: w \in \mathbb{D}, \eta \in \mathcal{E}\right\}$ is a total set in $A_{\alpha}^{2}(\mathcal{E})$ and $\left\langle f, B_{\alpha}(\cdot, w) \eta\right\rangle_{A_{\alpha}^{2}(\mathcal{E})}=$ $\langle f(w), \eta\rangle_{\mathcal{E}}$ where $f \in A_{\alpha}^{2}(\mathcal{E}), w \in \mathbb{D}$ and $\eta \in \mathcal{E}$. Moreover, it is easy to see that the shift operator $M_{z}$ on $A_{\alpha}^{2}(\mathcal{E}), \alpha \geq 1$, is a $C_{\cdot 0}$-contraction where

$$
\left(M_{z} f\right)(w)=w f(w) \quad\left(f \in A_{\alpha}^{2}(\mathcal{E}), w \in \mathbb{D}\right) .
$$

In the following discussion, we shall mostly use weighted Bergman spaces with only integer weights. Let us point out an important special case: $A_{1}^{2}=H^{2}$, the Hardy space over $\mathbb{D}$.

For a multi-index $\boldsymbol{m}=\left(m_{1}, \ldots, m_{n}\right) \in \mathbb{N}^{n}$ we denote the corresponding weighted Bergman space on $\mathbb{D}^{n}$ by $A_{\boldsymbol{m}}^{2}$. The weighted Bergman kernel on $\mathbb{D}^{n}$ with weight $\boldsymbol{m}$ is, by definition, the reproducing kernel function

$$
B_{\boldsymbol{m}}(\boldsymbol{z}, \boldsymbol{w})=\prod_{i=1}^{n} B_{m_{i}}\left(z_{i}, w_{i}\right)=\prod_{i=1}^{n}\left(1-z_{i} \bar{w}_{i}\right)^{-m_{i}} \quad\left(\boldsymbol{z}, \boldsymbol{w} \in \mathbb{D}^{n}\right) .
$$

For each $\boldsymbol{w} \in \mathbb{D}^{n}$, we denote by $B_{\boldsymbol{m}}(\cdot, \boldsymbol{w})$ the kernel function at $\boldsymbol{w}$, where

$$
\left(B_{\boldsymbol{m}}(\cdot, \boldsymbol{w})\right)(\boldsymbol{z})=B_{\boldsymbol{m}}(\boldsymbol{z}, \boldsymbol{w}) \quad\left(\boldsymbol{z} \in \mathbb{D}^{n}\right) .
$$

Convention: Let $p(\boldsymbol{z}, \boldsymbol{w})=\sum_{\boldsymbol{p}, \boldsymbol{q} \in \mathbb{N}^{n}} a_{\boldsymbol{p q}} \boldsymbol{z}^{\boldsymbol{p}} \overline{\boldsymbol{w}}^{\boldsymbol{q}}$ be a polynomial in $\left\{z_{1}, \ldots, z_{n}\right\}$ and $\left\{\bar{w}_{1}, \ldots, \bar{w}_{n}\right\}$. For a commuting tuple of bounded linear operators $\boldsymbol{T}=\left(T_{1}, \ldots, T_{n}\right)$ on a Hilbert space $\mathcal{H}$ (that is, $T_{i} T_{j}=T_{j} T_{i}$ for all $\left.i, j=1, \ldots, n\right)$ we denote by $p(\boldsymbol{z}, \boldsymbol{w})\left(\boldsymbol{T}, \boldsymbol{T}^{*}\right)$ the corresponding hereditary functional calculus in the sense of Agler [1], that is,

$$
p(z, w)\left(\boldsymbol{T}, \boldsymbol{T}^{*}\right)=\sum_{\boldsymbol{p}, \boldsymbol{q} \in \mathbb{N}^{n}} a_{\boldsymbol{p q}} \boldsymbol{T}^{p} \boldsymbol{T}^{* \boldsymbol{q}},
$$

where $\boldsymbol{T}^{\boldsymbol{k}}=T^{k_{1}} \cdots T_{n}^{k_{n}}$ and $\boldsymbol{T}^{* \boldsymbol{k}}=T_{1}^{* k-1} \cdots T_{n}^{* k_{n}}$ for all $\boldsymbol{k}=\left(k_{1}, \ldots, k_{n}\right) \in \mathbb{N}^{n}$.

Definition 2.1. A bounded linear operator $T$ on $\mathcal{H}$ is said to be $B_{m}$-contractive (or $T$ is a $B_{m}$-contraction) if $T$ is in $C_{.0}$ class and

$$
\begin{aligned}
B_{m}^{-1}(z, w)\left(T, T^{*}\right) & =\left(\sum_{k=0}^{m}(-1)^{k}\left(\begin{array}{c}
m \\
k
\end{array}\right) z^{k} \bar{w}^{* k}\right)\left(T, T^{*}\right) \\
& =\sum_{k=0}^{m}(-1)^{k}\left(\begin{array}{c}
m \\
k
\end{array}\right) T^{k} T^{* k} \geq 0 .
\end{aligned}
$$


We also recall that $T \in \mathcal{B}(\mathcal{H})$ is a hypercontraction of order $m$ [1] if

$$
B_{p}^{-1}(z, w)\left(T, T^{*}\right) \geq 0
$$

holds for all $1 \leq p \leq m$.

Now let $T$ be a $B_{m}$-contraction on $\mathcal{H}$. Since $T \in C_{.0}$, it follows from Lemma 2.11 in [1] that

$$
B_{p}^{-1}(z, w)\left(T, T^{*}\right) \geq 0 \quad(1 \leq p \leq m),
$$

that is, $T$ is a hypercontraction of order $m$. In other wards, these two notions coincide for $C_{.0}$ class of operators and hence, we will restrict our considerations for $B_{m}$-contractions.

The defect operator and the defect space of a $B_{m}$-contraction $T \in \mathcal{B}(\mathcal{H})$ are defined by

$$
D_{m, T}=\left(B_{m}^{-1}(z, w)\left(T, T^{*}\right)\right)^{\frac{1}{2}}, \quad \mathcal{D}_{m, T}=\overline{\operatorname{ran}} D_{m, T}
$$

respectively. Let us set

$$
B_{m}(z, T)=\left(I_{\mathcal{H}}-z T^{*}\right)^{-m} \quad(z \in \mathbb{D})
$$

and

$$
\left(\boldsymbol{v}_{m, T} f\right)(z)=D_{m, T} B_{m}(z, T) f=D_{m, T}\left(I_{\mathcal{H}}-z T^{*}\right)^{-m} f \quad(f \in \mathcal{H}, z \in \mathbb{D}) .
$$

Then $\boldsymbol{v}_{m, T}: \mathcal{H} \rightarrow A_{m}^{2}\left(\mathcal{D}_{m, T}\right)$ is a bounded linear operator,

$$
\boldsymbol{v}_{m, T} T^{*}=M_{z}^{*} \boldsymbol{v}_{m, T}
$$

and

$$
\boldsymbol{v}_{m, T}^{*}\left(B_{m}(\cdot, w) \eta\right)=B_{m}(w, T)^{*} D_{m, T} \eta=\left(I_{\mathcal{H}}-\bar{w} T\right)^{-m} D_{m, T} \eta,
$$

for all $w \in \mathbb{D}$ and $\eta \in \mathcal{D}_{m, T}$. This and the definition of $\boldsymbol{v}_{m, T}$ together imply

$$
\left(\boldsymbol{v}_{m, T} \boldsymbol{v}_{m, T}^{*}\left(B_{m}(\cdot, w) \eta\right)\right)(z)=D_{m, T} B_{m}(z, T) B_{m}(w, T)^{*} D_{m, T} \eta \quad(z \in \mathbb{D}),
$$

for all $w \in \mathbb{D}$ and $\eta \in \mathcal{D}_{m, T}$. Furthermore, if $T$ is a $B_{m}$-contraction then $\boldsymbol{v}_{m, T}$ is an isometry and hence a dilation of $T$ (see Agler [1]).

Theorem 2.2. (Agler) Let $T \in \mathcal{B}(\mathcal{H})$ be a $B_{m}$-contraction. Then $\left.T \cong P_{\mathcal{Q}} M_{z}\right|_{\mathcal{Q}}$, for some $M_{z}^{*}$-invariant closed subspace $\mathcal{Q}$ of $A_{m}^{2}\left(\mathcal{D}_{m, T}\right)$.

We shall now introduce the notion of multipliers on weighted Bergman spaces. Let $m_{1}, m_{2}$ be two natural numbers and $\mathcal{E}_{1}, \mathcal{E}_{2}$ be two Hilbert spaces. An operator valued holomorphic map $\Theta: \mathbb{D} \rightarrow \mathcal{B}\left(\mathcal{E}_{1}, \mathcal{E}_{2}\right)$ is said to be a multiplier from $A_{m_{1}}^{2}\left(\mathcal{E}_{1}\right)$ to $A_{m_{2}}^{2}\left(\mathcal{E}_{2}\right)$ if $\Theta f \in A_{m_{2}}^{2}\left(\mathcal{E}_{2}\right)$ for all $f \in A_{m_{1}}^{2}\left(\mathcal{E}_{1}\right)$. We denote the set of all multipliers from $A_{m_{1}}^{2}\left(\mathcal{E}_{1}\right)$ to $A_{m_{2}}^{2}\left(\mathcal{E}_{2}\right)$ by $\mathcal{M}\left(A_{m_{1}}^{2}\left(\mathcal{E}_{1}\right), A_{m_{2}}^{2}\left(\mathcal{E}_{2}\right)\right)$. We also use the notation $M_{\Theta}$, for each $\Theta \in \mathcal{M}\left(A_{m_{1}}^{2}\left(\mathcal{E}_{1}\right), A_{m_{2}}^{2}\left(\mathcal{E}_{2}\right)\right)$, to denote the multiplication operator

$$
M_{\Theta} f=\Theta f \quad\left(f \in A_{m_{1}}^{2}\left(\mathcal{E}_{1}\right)\right) .
$$

A multiplier $\Theta \in \mathcal{M}\left(A_{m_{1}}^{2}\left(\mathcal{E}_{1}\right), A_{m_{2}}^{2}\left(\mathcal{E}_{2}\right)\right)$ is said to be a partially isometric multiplier if $M_{\Theta}$ is a partially isometric operator from $A_{m_{1}}^{2}\left(\mathcal{E}_{1}\right)$ to $A_{m_{2}}^{2}\left(\mathcal{E}_{2}\right)$. 
Before proceeding, let us for the sake of completeness recall a Beurling-Lax-Halmos type theorem for weighted Bergman shifts (see [8] and Theorem 2.3 in [15]) upon which much of our discussion in this paper will rest.

TheOREM 2.3. Let $\mathcal{S}$ be a non-trivial closed subspace of $A_{m}^{2}\left(\mathcal{E}_{*}\right)$. Then $\mathcal{S}$ is $M_{z}$-invariant if and only if there exists a Hilbert space $\mathcal{E}$ and a partially isometric multiplier $\theta \in \mathcal{M}\left(A_{1}^{2}(\mathcal{E}), A_{m}^{2}\left(\mathcal{E}_{*}\right)\right)$ such that $\mathcal{S}=\theta A_{1}^{2}(\mathcal{E})$.

We are now ready to present a functional model for the class of $B_{m}$-contractions.

Theorem 2.4. Let $T \in \mathcal{B}(\mathcal{H})$ be a $B_{m}$-contraction. Then there exists a Hilbert space $\mathcal{E}$ and a partially isometric multiplier $\theta \in \mathcal{M}\left(A_{1}^{2}(\mathcal{E}), A_{m}^{2}\left(\mathcal{D}_{m, T}\right)\right)$ such that

$$
\left.T \cong P_{\mathcal{Q}_{\theta}} M_{z}\right|_{\mathcal{Q}_{\theta}}
$$

where $\mathcal{Q}_{\theta}=A_{m}^{2}\left(\mathcal{D}_{m, T}\right) \ominus \theta A_{1}^{2}(\mathcal{E})$.

Proof. At first, by virtue of Theorem 2.2, we realize $T$ as $\left.T \cong P_{\mathcal{Q}} M_{z}\right|_{\mathcal{Q}}$. Therefore, it only remains to prove the existence of a partially isometric multiplier $\theta$ such that $\mathcal{Q}=$ $A_{m}^{2}\left(\mathcal{D}_{m, T}\right) \ominus \theta A_{1}^{2}(\mathcal{E})$.

Note that since $\mathcal{Q}=\operatorname{ran} \boldsymbol{v}_{m, T}$ is $M_{z}^{*}$-invariant, $\left(\operatorname{ran} \boldsymbol{v}_{m, T}\right)^{\perp}$ is a $M_{z}$-invariant closed subspace of $A_{m}^{2}\left(\mathcal{D}_{m, T}\right)$. Then, applying Theorem 2.3 to $\left(\operatorname{ran} \boldsymbol{v}_{m, T}\right)^{\perp}$, we obtain a coefficient Hilbert space $\mathcal{E}$ and a partially isometric multiplier $\theta \in \mathcal{M}\left(A_{1}^{2}(\mathcal{E}), A_{m}^{2}\left(\mathcal{D}_{m, T}\right)\right)$ such that

$$
\left(\operatorname{ran} \boldsymbol{v}_{m, T}\right)^{\perp}=\theta A_{1}^{2}(\mathcal{E})
$$

that is,

$$
\mathcal{Q}=A_{m}^{2}\left(\mathcal{D}_{m, T}\right) \ominus \theta A_{1}^{2}(\mathcal{E})
$$

This completes the proof.

The following observation was pointed out to us by R. G. Douglas: Let $T \in \mathcal{B}(\mathcal{H})$ be a $B_{m}$-contraction. Then by Theorem 2.4 , we have

$$
H^{2}(\mathcal{E}) \stackrel{M_{\theta}}{\longrightarrow} A_{m}^{2}\left(\mathcal{D}_{m, T}\right) \stackrel{\pi}{\longrightarrow} \mathcal{H} \longrightarrow 0
$$

where $\pi=\boldsymbol{v}_{m, T}^{*}\left(\right.$ recall that $\left.A_{1}^{2}(\mathcal{E})=H^{2}(\mathcal{E})\right)$. Note that since $M_{\theta} M_{z}=M_{z} M_{\theta}, \mathcal{S}:=\operatorname{ker} M_{\theta}$ is a $M_{z}$-invariant subspace of $H^{2}(\mathcal{E})$. Then by Beurling-Lax-Halmos theorem there exists a Hilbert space $\mathcal{E}_{*}$ and an inner (or isometric) multiplier $\psi \in H_{\mathcal{B}\left(\mathcal{E}_{*}, \mathcal{E}\right)}^{\infty}(\mathbb{D})$ such that $\mathcal{S}=\psi H^{2}\left(\mathcal{E}_{*}\right)$. Consequently, we have a natural chain complex of Hilbert spaces:

$$
0 \longrightarrow H^{2}\left(\mathcal{E}_{*}\right) \stackrel{M_{\psi}}{\longrightarrow} H^{2}(\mathcal{E}) \stackrel{M_{\theta}}{\longrightarrow} A_{m}^{2}\left(\mathcal{D}_{m, T}\right) \stackrel{\pi}{\longrightarrow} \mathcal{H} \longrightarrow 0
$$

\section{Dilations of Commuting hypercontractions}

In this section, we give a proof of the fact that a doubly commuting tuple of hypercontractions can be dilated to the tuple of multiplication operators on a suitable weighted Bergman space over $\mathbb{D}^{n}$ (see $\left.[2,3,4,5]\right)$. We begin with a definition. 
Definition 3.1. A commuting tuple of operators $\boldsymbol{T}=\left(T_{1}, \ldots, T_{n}\right)$ on $\mathcal{H}$ is said to be $B_{\boldsymbol{m}^{-}}$ contractive if $T_{i}$ is a $B_{m_{i}}$-contraction, $i=1, \ldots, n$, and

$$
B_{\boldsymbol{m}}^{-1}(\boldsymbol{z}, \boldsymbol{w})\left(\boldsymbol{T}, \boldsymbol{T}^{*}\right)=\left(\prod_{i=1}^{n} B_{m_{i}}^{-1}\left(z_{i}, w_{i}\right)\right)\left(\boldsymbol{T}, \boldsymbol{T}^{*}\right) \geq 0 .
$$

We denote the (joint-) defect operator and defect space of a $B_{\boldsymbol{m}}$-contraction $\boldsymbol{T}$ as:

$$
D_{\boldsymbol{m}, \boldsymbol{T}}:=\left(B_{\boldsymbol{m}}^{-1}(\boldsymbol{z}, \boldsymbol{w})\left(\boldsymbol{T}, \boldsymbol{T}^{*}\right)\right)^{\frac{1}{2}}, \quad \text { and } \quad \mathcal{D}_{\boldsymbol{m}, \boldsymbol{T}}=\overline{\operatorname{ran}} D_{\boldsymbol{m}, \boldsymbol{T}},
$$

respectively.

For the rest of the paper we shall be dealing with a fixed natural number $n \geq 2$, and a multi-index $\boldsymbol{m}=\left(m_{1}, \ldots, m_{n}\right) \in \mathbb{N}^{n}, m_{j} \geq 1, j=1, \ldots, n$.

Let $\boldsymbol{T}$ be a doubly commuting $B_{m}$-contractive tuple on $\mathcal{H}$. Then

$$
T_{i}\left(B_{m_{j}}^{-1}(z, w)\left(T_{j}, T_{j}^{*}\right)\right)=\left(B_{m_{j}}^{-1}(z, w)\left(T_{j}, T_{j}^{*}\right)\right) T_{i},
$$

for all $i \neq j$. This also implies that

$$
\left(B_{m_{i}}^{-1}\left(z_{i}, w_{i}\right)\left(T_{i}, T_{i}^{*}\right)\right)\left(B_{m_{j}}^{-1}\left(z_{j}, w_{j}\right)\left(T_{j}, T_{j}^{*}\right)\right)=\left(B_{m_{j}}^{-1}\left(z_{j}, w_{j}\right)\left(T_{j}, T_{j}^{*}\right)\right)\left(B_{m_{i}}^{-1}\left(z_{i}, w_{i}\right)\left(T_{i}, T_{i}^{*}\right)\right) .
$$

The above observations yield the following:

LEMma 3.2. Let $\boldsymbol{T}$ be an n-tuple of doubly commuting operators on $\mathcal{H}$ and each $T_{i}$ is a $B_{m_{i}}$-contraction, $i=1, \ldots, n$. Then $\mathcal{D}_{m_{j}, T_{j}}$ is a $T_{i}$-reducing subspace of $\mathcal{H}$ and

$$
T_{i} D_{m_{j}, T_{j}}=D_{m_{j}, T_{j}} T_{i}, \quad D_{m_{j}, T_{j}} D_{m_{i}, T_{i}}=D_{m_{i}, T_{i}} D_{m_{j}, T_{j}},
$$

for all $i \neq j$. Moreover, $\boldsymbol{T}$ is a $B_{\boldsymbol{m}_{\text {-contraction }} \text { and }}$

$$
D_{\boldsymbol{m}, \boldsymbol{T}}=\prod_{i=1}^{n} D_{m_{i}, T_{i}}
$$

Now, we shall construct, using induction, a dilation map for a doubly commuting $B_{m^{-}}$ contractive tuple $\boldsymbol{T}$ on $\mathcal{H}$.

In what follows, for each $j \in\{2, \ldots, n\}, \boldsymbol{m}_{\boldsymbol{j}}$ denotes the $j$-tuple $\left(m_{1}, \ldots, m_{j}\right) \in \mathbb{N}^{j}$ and $\boldsymbol{T}_{\boldsymbol{j}}$ the doubly commuting $B_{\boldsymbol{m}_{\boldsymbol{j}}}$-contraction $\left(T_{1}, \ldots, T_{j}\right)$ on $\mathcal{H}$. For each $j \in\{2, \ldots, n\}$, one checks easily that $\mathcal{D}_{\boldsymbol{m}_{\boldsymbol{j}-1}, \boldsymbol{T}_{\boldsymbol{j}-1}}$ is a $T_{j}$-reducing closed subspace of $\mathcal{H}$ and

$$
D_{\left.T_{j}\right|_{\mathcal{D}_{m_{j-1}, T_{j-1}}}}=\left.D_{T_{j}}\right|_{\mathcal{D}_{m_{j-1}, T_{j-1}}}, \quad \text { and } \quad \mathcal{D}_{\left.T_{j}\right|_{\mathcal{D}_{\boldsymbol{m}_{j-1}, T_{j-1}}}}=\mathcal{D}_{\boldsymbol{m}_{\boldsymbol{j}}, \boldsymbol{T}_{\boldsymbol{j}}},
$$

where $\mathcal{D}_{\boldsymbol{m}_{\mathbf{1}}, \boldsymbol{T}_{\mathbf{1}}}=\mathcal{D}_{m_{1}, T_{1}}$. Now we set $V_{1}:=\boldsymbol{v}_{m_{1}, T_{1}}: \mathcal{H} \rightarrow A_{m_{1}}^{2}\left(\mathcal{D}_{m_{1}, T_{1}}\right)$ and define

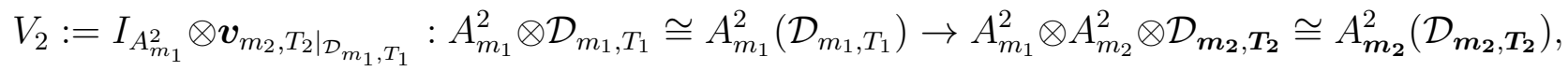

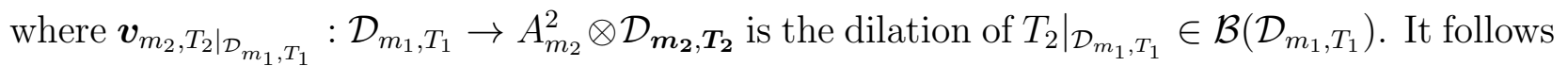
that, for all $l \in \mathbb{N}, h \in \mathcal{D}_{m_{1}, T_{1}}$,

$$
\left(V_{2}\left(z^{l} h\right)\right)\left(z_{1}, z_{2}\right)=z_{1}^{l}\left(\boldsymbol{v}_{m_{2}, T_{2}} h\right)\left(z_{2}\right) .
$$


Continuing this way, one can construct $n$ bounded linear operators $\left\{V_{j}\right\}_{j=2}^{n}$ defined by

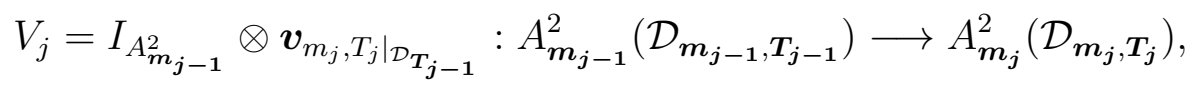

where

$$
\left(V_{j}\left(z_{1}^{k_{1}} \cdots z_{j-1}^{k_{j-1}} h\right)\right)\left(z_{1}, \ldots, z_{j}\right)=z_{1}^{k_{1}} \cdots z_{j-1}^{k_{j-1}}\left(\boldsymbol{v}_{m_{j},\left.T_{j}\right|_{\mathcal{D}_{\boldsymbol{m}_{j-1}, \boldsymbol{T}_{j-1}}}} h\right)\left(z_{j}\right)
$$

for all $h \in \mathcal{D}_{\boldsymbol{m}_{\boldsymbol{j}-1}, \boldsymbol{T}_{\boldsymbol{j}-1}}$ and $j=2, \ldots, n$. Consequently, we have the following sequence of maps:

$$
0 \longrightarrow \mathcal{H} \stackrel{V_{1}}{\longrightarrow} A_{m_{1}}^{2}\left(\mathcal{D}_{m_{1}, T_{1}}\right) \stackrel{V_{2}}{\longrightarrow} A_{\boldsymbol{m}_{\mathbf{2}}}^{2}\left(\mathcal{D}_{\boldsymbol{m}_{\mathbf{2}}, \boldsymbol{T}_{\mathbf{2}}}\right) \stackrel{V_{3}}{\longrightarrow} \cdots \stackrel{V_{n}}{\longrightarrow} A_{\boldsymbol{m}}^{2}\left(\mathcal{D}_{\boldsymbol{m}, \boldsymbol{T}}\right)
$$

Let us denote by $V_{\boldsymbol{T}}$ the compositions of $\left\{V_{j}\right\}_{j=1}^{n}$ :

$$
V_{\boldsymbol{T}}:=V_{n} \circ \cdots \circ V_{2} \circ V_{1}: \mathcal{H} \rightarrow A_{\boldsymbol{m}}^{2}\left(\mathcal{D}_{\boldsymbol{m}, \boldsymbol{T}}\right) .
$$

Then $V_{\boldsymbol{T}} \in \mathcal{B}\left(\mathcal{H}, A_{\boldsymbol{m}}^{2}\left(\mathcal{D}_{\boldsymbol{m}, \boldsymbol{T}}\right)\right)$ is an isometric dilation of $\boldsymbol{T}$ :

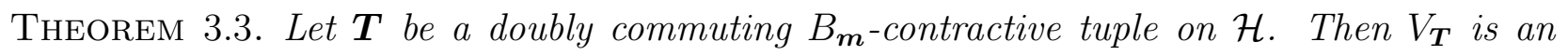
isometry and

$$
\left(V_{\boldsymbol{T}} h\right)(\boldsymbol{z})=\left(\prod_{i=1}^{n} D_{m_{i}, T_{i}} B_{m_{i}}\left(z_{i}, T_{i}\right)\right) h \quad\left(h \in \mathcal{H}, \boldsymbol{z} \in \mathbb{D}^{n}\right) .
$$

Moreover, $V_{\boldsymbol{T}} T_{i}^{*}=M_{z_{i}}^{*} V_{\boldsymbol{T}}, i=1, \ldots, n$, and for each $\boldsymbol{w} \in \mathbb{D}^{n}$ and $\eta \in \mathcal{D}_{\boldsymbol{m}, \boldsymbol{T}}$,

$$
\left(\left(V_{\boldsymbol{T}} V_{\boldsymbol{T}}^{*}\right)\left(B_{\boldsymbol{m}}(\cdot, \boldsymbol{w}) \eta\right)\right)(\boldsymbol{z})=\prod_{i=1}^{n} D_{m_{i}, T_{i}} B_{m_{i}}\left(z_{i}, T_{i}\right) B_{m_{i}}\left(w_{i}, T_{i}\right)^{*} D_{m_{i}, T_{i}} \eta . \quad\left(\boldsymbol{z} \in \mathbb{D}^{n}\right)
$$

Proof. Clearly $V_{1}^{*} V_{1}=I_{\mathcal{H}}$ and for each $j=2, \ldots, n$, we have

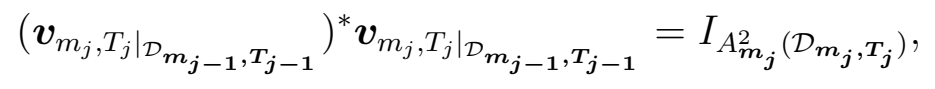

from which we immediately deduce $V_{j}^{*} V_{j}=I_{A_{m_{j-1}}^{2}}\left(\mathcal{D}_{m_{j-1}, T_{j-1}}\right)$, and finally $V_{\boldsymbol{T}}^{*} V_{\boldsymbol{T}}=I_{\mathcal{H}}$. Now by (3.1), we have

$$
\begin{aligned}
V_{\boldsymbol{T}} h & =V_{n} \cdots V_{2}\left(V_{1} h\right)=V_{n} \cdots V_{3}\left(V_{2} D_{m_{1}, T_{1}} B_{m_{1}}\left(z_{1}, T_{1}\right) h\right) \\
& =V_{n} \cdots V_{3}\left(D_{m_{2}, T_{2}} B_{m_{2}}\left(z_{2}, T_{2}\right) D_{m_{1}, T_{1}} B_{m_{1}}\left(z_{1}, T_{1}\right) h\right) \\
& =V_{n} \cdots V_{3}\left(D_{m_{1}, T_{1}} D_{m_{2}, T_{2}} B_{m_{1}}\left(z_{1}, T_{1}\right) B_{m_{2}}\left(z_{2}, T_{2}\right) h\right),
\end{aligned}
$$

for all $h \in \mathcal{H}$. Continuing this way we have

$$
\left(V_{\boldsymbol{T}} h\right)(\boldsymbol{z})=\prod_{i=1}^{n} D_{m_{i}, T_{i}} B_{m_{i}}\left(z_{i}, T_{i}\right) h \quad\left(h \in \mathcal{H}, \boldsymbol{z} \in \mathbb{D}^{n}\right) .
$$

This and a direct computation (or see [2] or [11]) readily implies the intertwining property of $V_{\boldsymbol{T}}$ and

$$
V_{\boldsymbol{T}}^{*}\left(B_{\boldsymbol{m}}(\cdot, \boldsymbol{w}) \eta\right)=\prod_{i=1}^{n} B_{m_{i}}\left(w_{i}, T_{i}\right)^{*} D_{m_{i}, T_{i}} \eta \quad\left(\boldsymbol{w} \in \mathbb{D}^{n}, \eta \in \mathcal{D}_{\boldsymbol{m}, \boldsymbol{T}}\right) .
$$

which in turn yields the last part of the theorem. This completes the proof. 
The above theorem is a doubly commuting version and a particular case of Theorem 3.16 in [11] by Curto and Vasilescu and Corollary 16 in [2] by Ambrozie, Englis and Muller (see also [9]). However, the present approach is based on the idea of "simple tensor products of one variable dilation maps". Moreover, our construction of explicit dilation map is especially useful in analytic model theory (see Section 4).

Recall that a pair of commuting tuples $\boldsymbol{T}=\left(T_{1}, \ldots, T_{n}\right)$ on $\mathcal{H}$ and $\boldsymbol{S}=\left(S_{1}, \ldots, S_{n}\right)$ on $\mathcal{K}$ is said to be jointly unitarily equivalent, also denoted by $\boldsymbol{T} \cong \boldsymbol{S}$, if there exists a unitary map $U: \mathcal{H} \rightarrow \mathcal{K}$ such that $U T_{i}=S_{i} U, i=1, \ldots, n$. The following dilation result is an easy consequence of Theorem 3.3.

TheOREM 3.4. Let $\boldsymbol{T}$ be a doubly commuting $B_{\boldsymbol{m}}$-contractive tuple on $\mathcal{H}$. Then there exists a joint $\left(M_{z_{1}}^{*}, \ldots, M_{z_{n}}^{*}\right)$-invariant closed subspace $\mathcal{Q} \subseteq A_{m}^{2}\left(\mathcal{D}_{\mathcal{D}_{m, T}}\right)$ such that

$$
\left(T_{1}, \ldots, T_{n}\right) \cong\left(\left.P_{\mathcal{Q}} M_{z_{1}}\right|_{\mathcal{Q}}, \ldots,\left.P_{\mathcal{Q}} M_{z_{n}}\right|_{\mathcal{Q}}\right)
$$

Proof. Let $\mathcal{Q}=\operatorname{ran} V_{\boldsymbol{T}}$, where $V_{\boldsymbol{T}}$ is the dilation map of $T$ as in Theorem 3.3. Then $\mathcal{Q}$ is a joint $\left(M_{z_{1}}^{*}, \ldots, M_{z_{n}}^{*}\right)$-invariant subspace of $A_{\boldsymbol{m}}^{2}(\mathcal{H})$ and $U_{\boldsymbol{T}}:=V_{\boldsymbol{T}}: \mathcal{H} \rightarrow \mathcal{Q}$ is a unitary map. Moreover,

$$
U_{\boldsymbol{T}} T_{j}^{*}=V_{\boldsymbol{T}} T_{j}^{*}=M_{z_{j}}^{*} V_{\boldsymbol{T}}=M_{z_{j}}^{*} V_{\boldsymbol{T}} V_{\boldsymbol{T}}^{*} V_{\boldsymbol{T}}=\left(\left.M_{z_{j}}^{*}\right|_{\mathcal{Q}}\right) V_{\boldsymbol{T}}=\left(\left.M_{z_{j}}^{*}\right|_{\mathcal{Q}}\right) U_{\boldsymbol{T}},
$$

for all $j=1, \ldots, n$. Hence

$$
U_{\boldsymbol{T}} T_{j}=\left.P_{\mathcal{Q}} M_{z_{j}}\right|_{\mathcal{Q}} U_{\boldsymbol{T}} \quad(j=1, \ldots, n) .
$$

This completes the proof of the theorem.

\section{Analytic model}

We begin with the following lemma, the relevance of which to our purpose will become apparent in connection with the analytic model of doubly commuting tuples of operators.

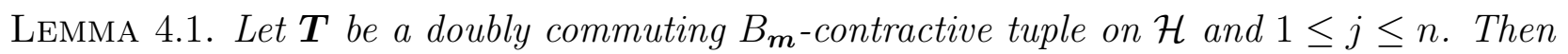
$A_{\boldsymbol{m}}^{2}\left(\mathcal{D}_{\boldsymbol{m}, \boldsymbol{T}}\right) \subseteq A_{\boldsymbol{m}}^{2}\left(\mathcal{D}_{m_{j}, T_{j}}\right)$ and $A_{\boldsymbol{m}}^{2}\left(\mathcal{D}_{\boldsymbol{m}, \boldsymbol{T}}\right)$ is a reducing subspace for

$$
\left(\bigotimes_{\substack{i=1 \\ i \neq j}}^{n} I_{A_{m_{i}}^{2}}\right) \otimes \boldsymbol{v}_{m_{j}, T_{j}} \boldsymbol{v}_{m_{j}, T_{j}}^{*} \in \mathcal{B}\left(A_{\boldsymbol{m}}^{2}\left(\mathcal{D}_{m_{j}, T_{j}}\right)\right) .
$$

Proof. The first part follows from the fact that $\mathcal{D}_{\boldsymbol{m}, \boldsymbol{T}} \subseteq \mathcal{D}_{m_{j}, T_{j}}$. For the second part, it is enough to prove that $X_{j}\left(B_{\boldsymbol{m}}(\cdot, \boldsymbol{w}) \eta\right) \in A_{\boldsymbol{m}}^{2}\left(\mathcal{D}_{\boldsymbol{m}, \boldsymbol{T}}\right)$ where $\boldsymbol{w} \in \mathbb{D}^{n}, \eta \in \mathcal{D}_{\boldsymbol{m}, \boldsymbol{T}}$ and

$$
X_{j}:=\left(\bigotimes_{\substack{i=1 \\ i \neq j}}^{n} I_{A_{m_{i}}^{2}}\right) \otimes \boldsymbol{v}_{m_{j}, T_{j}} \boldsymbol{v}_{m_{j}, T_{j}}^{*} .
$$


To this end, for each $z_{j}, w_{j} \in \mathbb{D}$, we compute

$$
\begin{aligned}
D_{m_{j}, T_{j}} B_{m_{j}}\left(z_{j}, T_{j}\right) B_{m_{j}}\left(w_{j}, T_{j}\right)^{*} D_{m_{j}, T_{j}}\left(\prod_{i=1}^{n} D_{m_{i}, T_{i}}\right) \\
=D_{m_{j}, T_{j}} B_{m_{j}}\left(z_{j}, T_{j}\right) B_{m_{j}}\left(w_{j}, T_{j}\right)^{*}\left(\prod_{\substack{i=1 \\
i \neq j}}^{n} D_{m_{i}, T_{i}}\right) D_{m_{j}, T_{j}}^{2} \\
=D_{m_{j}, T_{j}}\left(\prod_{\substack{i=1 \\
i \neq j}}^{n} D_{m_{i}, T_{i}}\right) B_{m_{j}}\left(z_{j}, T_{j}\right) B_{m_{j}}\left(w_{j}, T_{j}\right)^{*} D_{m_{j}, T_{j}}^{2} \\
=\left(\prod_{i=1}^{n} D_{m_{i}, T_{i}}\right) B_{m_{j}}\left(z_{j}, T_{j}\right) B_{m_{j}}\left(w_{j}, T_{j}\right)^{*} D_{m_{j}, T_{j}}^{2} .
\end{aligned}
$$

In particular, we have

$$
\left(D_{m_{j}, T_{j}} B_{m_{j}}\left(z_{j}, T_{j}\right) B_{m_{j}}\left(w_{j}, T_{j}\right)^{*} D_{m_{j}, T_{j}}\right) \mathcal{D}_{\boldsymbol{m}, \boldsymbol{T}} \subseteq \mathcal{D}_{\boldsymbol{m}, \boldsymbol{T}} \quad\left(z_{j}, w_{j} \in \mathbb{D}\right) .
$$

If $\boldsymbol{w} \in \mathbb{D}^{n}, \eta=\prod_{i=1}^{n} D_{m_{i}, T_{i}} h \in \mathcal{D}_{\boldsymbol{m}, \boldsymbol{T}}$ and $h \in \mathcal{H}$, then (2.4) gives

$$
X_{j}\left(B_{\boldsymbol{m}}(\cdot, \boldsymbol{w}) \eta\right)=\left(\prod_{\substack{k=1 \\ k \neq j}}^{n} B_{m_{k}}\left(z_{k}, w_{k}\right)\right)\left(D_{m_{j}, T_{j}} B_{m_{j}}\left(z_{j}, T_{j}\right) B_{m_{j}}\left(w_{j}, T_{j}\right)^{*} D_{m_{j}, T_{j}} \eta\right) \in A_{\boldsymbol{m}}^{2}\left(\mathcal{D}_{\boldsymbol{m}, \boldsymbol{T}}\right) .
$$

This proves the desired claim, and the result follows.

Therefore, $R_{j} \in \mathcal{B}\left(A_{\boldsymbol{m}}^{2}\left(\mathcal{D}_{\boldsymbol{m}, \boldsymbol{T}}\right)\right), j=1, \ldots, n$, where

$$
R_{j}=\left.\left(\bigotimes_{\substack{i=1 \\ i \neq j}}^{n} I_{A_{m_{i}}^{2}}\right) \otimes\left(\boldsymbol{v}_{m_{j}, T_{j}} \boldsymbol{v}_{m_{j}, T_{j}}^{*}\right)\right|_{A_{m_{j}}^{2}\left(\mathcal{D}_{\boldsymbol{m}, \boldsymbol{T}}\right)}
$$

By virtue of (2.4) we have in particular for $\boldsymbol{w} \in \mathbb{D}^{n}, \eta \in \mathcal{D}_{\boldsymbol{m}, \boldsymbol{T}}$, and for $j=1, \ldots, n$ :

$$
R_{j}\left(B_{\boldsymbol{m}}(\cdot, \boldsymbol{w}) \eta\right)=\left(\prod_{\substack{i=1 \\ i \neq j}}^{n} B_{m_{i}}\left(z_{i}, w_{i}\right)\right)\left(D_{m_{j}, T_{j}} B_{m_{j}}\left(z_{j}, T_{j}\right) B_{m_{j}}\left(w_{j}, T_{j}\right)^{*} D_{m_{j}, T_{j}} \eta\right)
$$

Claim: $\left\{R_{1}, \ldots, R_{n}\right\}$ is a family of commuting orthogonal projections.

Proof of the claim: Since $\boldsymbol{v}_{m_{j}, T_{j}}$ is an isometry, we deduce from the definition of $R_{j}$ that $R_{j}=R_{j}^{*}=R_{j}^{2}, 1 \leq j \leq n$, that is, $\left\{R_{j}\right\}_{j=1}^{n}$ is a family of orthogonal projections. Now let 
$p, q \in \mathbb{N}, p \neq q, \boldsymbol{w} \in \mathbb{D}^{n}$ and $\eta \in \mathcal{D}_{\boldsymbol{m}, \boldsymbol{T}}$. Using (4.1), we obtain

$$
\begin{aligned}
R_{p} R_{q}\left(B_{\boldsymbol{m}}(\cdot, \boldsymbol{w}) \eta\right)= & R_{p}\left(\prod_{\substack{i=1 \\
i \neq q}}^{n} B_{m_{i}}\left(z_{i}, w_{i}\right)\left(D_{m_{q}, T_{q}} B_{m_{q}}\left(z_{q}, T_{q}\right) B_{m_{q}}\left(w_{q}, T_{q}\right)^{*} D_{m_{q}, T_{q}} \eta\right)\right) \\
= & \prod_{\substack{i=1 \\
i \neq p, q}}^{n} B_{m_{i}}\left(z_{i}, w_{i}\right)\left(D_{m_{p}, T_{p}} B_{m_{p}}\left(z_{p}, T_{p}\right) B_{m_{p}}\left(w_{p}, T_{p}\right)^{*} D_{m_{p}, T_{p}}\right) \\
& \left(D_{m_{q}, T_{q}} B_{m_{q}}\left(z_{q}, T_{q}\right) B_{m_{q}}\left(w_{q}, T_{q}\right)^{*} D_{m_{q}, T_{q}} \eta\right) \\
= & \prod_{\substack{i=1 \\
i \neq p, q}}^{n} B_{m_{i}}\left(z_{i}, w_{i}\right)\left(D_{m_{p}, T_{p}} D_{m_{q}, T_{q}} B_{m_{p}}\left(z_{p}, T_{p}\right) B_{m_{q}}\left(z_{q}, T_{q}\right)\right. \\
= & \left.B_{m_{p}}\left(w_{p}, T_{p}\right)^{*} B_{m_{q}}\left(w_{q}, T_{q}\right)^{*} D_{m_{p}, T_{p}} D_{m_{q}, T_{q}}\right) \eta \\
= & R_{q} R_{p}\left(B_{\boldsymbol{m}}(\cdot, \boldsymbol{w}) \eta\right) .
\end{aligned}
$$

Therefore $R_{p} R_{q}=R_{q} R_{p}$ for all $p, q=1, \ldots, n$. The proof of the claim is now complete.

We turn now to investigate the product $\prod_{j=1}^{n} R_{j}$. For sake of computational simplicity, let us assume, for each $i=1, \ldots, n$,

$$
f_{i}(z, w):=B_{m_{i}}\left(z, T_{i}\right) B_{m_{i}}\left(w, T_{i}\right)^{*} \quad(z, w \in \mathbb{D}) .
$$

For each $\boldsymbol{w} \in \mathbb{D}^{n}$ and $\eta \in \mathcal{D}_{\boldsymbol{m}, \boldsymbol{T}}$, we have

$$
\begin{aligned}
\left(\prod_{j=1}^{n} R_{j}\right)\left(B_{\boldsymbol{m}}(\cdot, \boldsymbol{w}) \eta\right) & =\prod_{\substack{j=1 \\
j \neq 1}}^{n} R_{j}\left(R_{1} B_{\boldsymbol{m}}(\cdot, \boldsymbol{w}) \eta\right) \\
& =\prod_{\substack{j=1 \\
j \neq 1}}^{n} R_{j}\left(\prod_{\substack{i=1 \\
i \neq 1}}^{n} B_{m_{i}}\left(\cdot, w_{i}\right) D_{m_{1}, T_{1}} f_{1}\left(\cdot, w_{1}\right) D_{m_{1}, T_{1}} \eta\right) \\
& =\prod_{\substack{j=1 \\
j \neq 1,2}}^{n} R_{j}\left(\prod_{\substack{i=1 \\
i \neq 1,2}}^{n} B_{m_{i}}\left(\cdot, w_{i}\right) D_{m_{1}, T_{1}} D_{m_{2}, T_{2}} f_{1}\left(\cdot, w_{1}\right) f_{2}\left(\cdot, w_{2}\right) D_{m_{1}, T_{1}} D_{m_{2}, T_{2}} \eta\right) .
\end{aligned}
$$

Continuing this way we have

$$
\left(\prod_{j=1}^{n} R_{j}\right)\left(B_{\boldsymbol{m}}(\cdot, \boldsymbol{w}) \eta\right)=\prod_{i=1}^{n} D_{m_{i}, T_{i}} f\left(\cdot, w_{i}\right) D_{m_{i}, T_{i}}=\prod_{i=1}^{n} D_{m_{i}, T_{i}} B_{m_{1}}\left(\cdot, T_{i}\right) B_{m_{i}}\left(w_{i}, T_{i}\right)^{*} D_{m_{i}, T_{i}},
$$

and hence Theorem 3.3 yields $V_{\boldsymbol{T}} V_{\boldsymbol{T}}^{*}=\prod_{i=1}^{n} R_{i}$. Summing up, we obtain the following:

THEOREM 4.2. Let $\boldsymbol{T}$ be a doubly commuting $B_{\boldsymbol{m}}$-contractive tuple on $\mathcal{H}$. Then $\left\{R_{i}\right\}_{i=1}^{n}$ is a family of commuting orthogonal projections and

$$
V_{\boldsymbol{T}} V_{\boldsymbol{T}}^{*}=\prod_{i=1}^{n} R_{i}
$$


We need to introduce one more notion. For $\boldsymbol{m} \in \mathbb{N}^{n}$ and for each $j=1, \ldots, n$, set

$$
\hat{\boldsymbol{m}}_{j}:=(m_{1}, \ldots, m_{j-1}, \underbrace{1}_{j-\text { th }}, m_{j+1}, \ldots, m_{n}) .
$$

In particular,

$$
A_{\hat{\boldsymbol{m}}_{j}}^{2}=A_{m_{1}}^{2} \otimes \cdots \otimes A_{m_{j-1}}^{2} \otimes H^{2} \otimes A_{m_{j+1}}^{2} \otimes \cdots \otimes A_{m_{n}}^{2}
$$

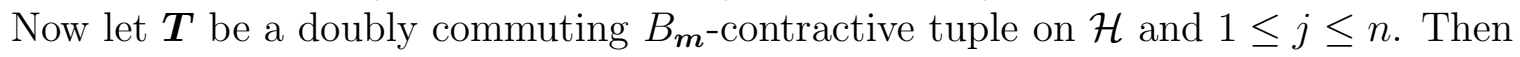

$$
\begin{aligned}
\operatorname{ran} R_{j} & =\left(\left(\bigotimes_{\substack{i=1 \\
i \neq j}}^{n} A_{m_{i}}^{2}\right) \otimes \operatorname{ran}\left(\boldsymbol{v}_{m_{j}, T_{j}} \boldsymbol{v}_{m_{j}, T_{j}}^{*}\right)\right) \bigcap\left(\left(\bigotimes_{\substack{i=1 \\
i \neq j}}^{n} A_{m_{i}}^{2}\right) \otimes A_{m_{j}}^{2}\left(\mathcal{D}_{\boldsymbol{m}, \boldsymbol{T}}\right)\right) \\
& =\left(\bigotimes_{\substack{i=1 \\
i \neq j}}^{n} A_{m_{i}}^{2}\right) \otimes \mathcal{Q}_{j},
\end{aligned}
$$

where $\mathcal{Q}_{j}=\operatorname{ran}\left(\boldsymbol{v}_{m_{j}, T_{j}} \boldsymbol{v}_{m_{j}, T_{j}}^{*}\right) \cap A_{m_{j}}^{2}\left(\mathcal{D}_{\boldsymbol{m}, \boldsymbol{T}}\right)$. But $\operatorname{ran}\left(\boldsymbol{v}_{m_{j}, T_{j}} \boldsymbol{v}_{m_{j}, T_{j}}^{*}\right)$ is an $M_{z}^{*}$-invariant subspace of $A_{m_{j}}^{2}\left(\mathcal{D}_{m_{j}, T_{j}}\right)$, hence $\mathcal{Q}_{j}$ is an $M_{z}^{*}$-invariant closed subspace of $A_{m_{j}}^{2}\left(\mathcal{D}_{\boldsymbol{m}, \boldsymbol{T}}\right)$. By Theorem 2.3 there exists an auxiliary Hilbert space $\mathcal{E}_{j}$ and a partially isometric multiplier $\theta_{j} \in \mathcal{M}\left(A_{1}^{2}\left(\mathcal{E}_{j}\right), A_{m_{j}}^{2}\left(\mathcal{D}_{\boldsymbol{m}, \boldsymbol{T}}\right)\right)$ such that

$$
R_{j}=I_{A_{\boldsymbol{m}}^{2}\left(\mathcal{D}_{\boldsymbol{m}, \boldsymbol{T}}\right)}-M_{\Theta_{j}} M_{\Theta_{j}}^{*}
$$

where

$$
M_{\Theta_{j}}=\left(\bigotimes_{\substack{i=1 \\ i \neq j}}^{n} I_{A_{m_{i}}^{2}}\right) \otimes M_{\theta_{j}} .
$$

Notice that $\Theta_{j} \in \mathcal{M}\left(A_{\hat{\boldsymbol{m}}_{j}}^{2}\left(\mathcal{E}_{j}\right), A_{\boldsymbol{m}}^{2}\left(\mathcal{D}_{\boldsymbol{m}, \boldsymbol{T}}\right)\right)$ is a partially isometric multiplier and

$$
\Theta_{j}(\boldsymbol{z})=\theta_{j}\left(z_{j}\right) \quad\left(\boldsymbol{z} \in \mathbb{D}^{n}\right) .
$$

This and Theorem 4.2 yields the following:

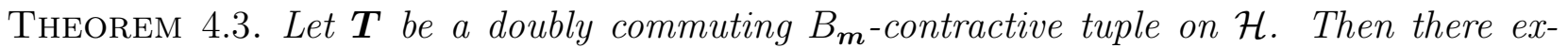
ists Hilbert spaces $\mathcal{E}_{k}$ and partially isometric multipliers $\theta_{k} \in \mathcal{M}\left(A_{1}^{2}\left(\mathcal{E}_{k}\right), A_{m_{k}}^{2}\left(\mathcal{D}_{\boldsymbol{m}, \boldsymbol{T}}\right)\right), k=$ $1, \ldots, n$, such that

$$
\left(M_{\Theta_{i}} M_{\Theta_{i}}^{*}\right)\left(M_{\Theta_{j}} M_{\Theta_{j}}^{*}\right)=\left(M_{\Theta_{j}} M_{\Theta_{j}}^{*}\right)\left(M_{\Theta_{i}} M_{\Theta_{i}}^{*}\right),
$$

for all $i, j, k=1, \ldots, n$. Moreover

$$
V_{\boldsymbol{T}} V_{\boldsymbol{T}}^{*}=\prod_{i=1}^{n}\left(I_{A_{\boldsymbol{m}}^{2}\left(\mathcal{D}_{\boldsymbol{m}, \boldsymbol{T}}\right)}-M_{\Theta_{i}} M_{\Theta_{i}}^{*}\right) .
$$

In order to formulate our functional model for $B_{m}$-contractive tuples, we need to recall the following result concerning commuting orthogonal projections (cf. Lemma 1.5 in [14]):

LEMMA 4.4. Let $\left\{P_{i}\right\}_{i=1}^{n}$ be a collection of commuting orthogonal projections on a Hilbert space $\mathcal{H}$. Then $\mathcal{L}:=\sum_{i=1}^{n}$ ranP $P_{i}$ is closed and the orthogonal projection of $\mathcal{H}$ onto $\mathcal{L}$ is given by $P_{\mathcal{L}}=I-\prod_{i=1}^{n}\left(I-P_{i}\right)$. 
We are now ready to present the main theorem of this section.

TheOREM 4.5. Let $\boldsymbol{T}$ be a doubly commuting $B_{m}$-contractive tuple on $\mathcal{H}$. Then there exists Hilbert spaces $\mathcal{E}_{k}$ and partial isometric multipliers $\theta_{k} \in \mathcal{M}\left(A_{1}^{2}\left(\mathcal{E}_{k}\right), A_{m_{k}}^{2}\left(\mathcal{D}_{\boldsymbol{m}, \boldsymbol{T}}\right)\right), k=1, \ldots, n$, such that

$$
\mathcal{H} \cong \mathcal{Q}_{\Theta}:=A_{\boldsymbol{m}}^{2}\left(\mathcal{D}_{\boldsymbol{m}, \boldsymbol{T}}\right) / \sum_{i=1}^{n} \Theta_{i} A_{\hat{\boldsymbol{m}}_{i}}^{2}\left(\mathcal{E}_{i}\right)
$$

and

$$
\left(T_{1}, \ldots, T_{n}\right) \cong\left(\left.P_{\mathcal{Q}_{\Theta}} M_{z_{1}}\right|_{\mathcal{Q}_{\Theta}}, \ldots,\left.P_{\mathcal{Q}_{\Theta}} M_{z_{n}}\right|_{\mathcal{Q}_{\Theta}}\right),
$$

where $\Theta_{i}$ is the one variable multiplier corresponding to $\theta_{i}, i=1, \ldots, n$, as defined in (4.2).

Proof. We continue with the notation of Theorem 4.3. Set $P_{i}:=M_{\Theta_{i}} M_{\Theta_{i}}^{*}, i=1, \ldots, n$. By virtue of Theorem 4.3 we have

$$
I_{A_{m}^{2}\left(\mathcal{D}_{\boldsymbol{m}, \boldsymbol{T}}\right)}-V_{\boldsymbol{T}} V_{\boldsymbol{T}}^{*}=I_{A_{\boldsymbol{m}}^{2}\left(\mathcal{D}_{\boldsymbol{m}, \boldsymbol{T}}\right)}-\prod_{i=1}^{n}\left(I_{A_{m}^{2}\left(\mathcal{D}_{\boldsymbol{m}, \boldsymbol{T}}\right)}-P_{i}\right) .
$$

Now by Lemma 4.4, it follows that

$$
\left(\operatorname{ran} V_{\boldsymbol{T}}\right)^{\perp}=\sum_{i=1}^{n} \operatorname{ran} M_{\Theta_{i}}=\sum_{i=1}^{n} \Theta_{i} A_{\hat{\boldsymbol{m}}_{i}}^{2}\left(\mathcal{E}_{i}\right) .
$$

Therefore,

$$
\mathcal{Q}_{\Theta}:=\operatorname{ran} V_{\boldsymbol{T}}=\left(\sum_{i=1}^{n} \Theta_{i} A_{\hat{\boldsymbol{m}}_{i}}^{2}\left(\mathcal{E}_{i}\right)\right)^{\perp} \cong A_{\boldsymbol{m}}^{2}\left(\mathcal{D}_{\mathcal{D}_{\boldsymbol{m}, \boldsymbol{T}}}\right) / \sum_{i=1}^{n} \Theta_{i} A_{\hat{\boldsymbol{m}}_{i}}^{2}\left(\mathcal{E}_{i}\right) .
$$

Now using the line of argument from the proof of Theorem 3.4 one can prove that $\left(T_{1}, \ldots, T_{n}\right) \cong$ $\left(\left.P_{\mathcal{Q}_{\Theta}} M_{z_{1}}\right|_{\mathcal{Q}_{\Theta}}, \ldots,\left.P_{\mathcal{Q}_{\Theta}} M_{z_{n}}\right|_{\mathcal{Q}_{\Theta}}\right)$. This concludes the proof.

In the special case that $\boldsymbol{m}=(1, \ldots, 1)$ we recover the functional model for doubly commuting tuples of pure contractions [9]. Moreover, the methods used here are different from those used in [9].

\section{Quotient modules of $A_{m}^{2}$}

We have a particular interest in tuples of operators $\left(M_{z_{1}}, \ldots, M_{z_{n}}\right)$ compressed to joint $\left(M_{z_{1}}^{*}, \ldots, M_{z_{n}}^{*}\right)$-invariant subspaces of reproducing kernel Hilbert spaces over $\mathbb{D}^{n}$. Let $\mathcal{Q}$ be a joint $\left(M_{z_{1}}^{*}, \ldots, M_{z_{n}}^{*}\right)$-invariant closed subspace of $A_{m}^{2}$ and $C_{z_{i}}=\left.P_{\mathcal{Q}} M_{z_{i}}\right|_{\mathcal{Q}}, i=1, \ldots, n$. Then $\mathcal{Q}$ is called a doubly commuting quotient module of $A_{m}^{2}$ if

$$
C_{z_{i}}^{*} C_{z_{j}}-C_{z_{j}} C_{z_{i}}^{*}=0 \quad(1 \leq i<j \leq n) .
$$

First, we compute the defect operator of a given doubly commuting quotient module $\mathcal{Q}$ of $A_{m}^{2}$ :

$$
D_{\boldsymbol{m}, \boldsymbol{C}_{\boldsymbol{z}}}^{2}=\prod_{i=1}^{n} B_{m_{i}}^{-1}\left(z_{i}, w_{i}\right)\left(C_{z}, C_{z}^{*}\right)=\left.P_{\mathcal{Q}}\left(\prod_{i=1}^{n} B_{m_{i}}^{-1}\left(M_{z_{i}}, M_{z_{i}}^{*}\right)\right)\right|_{\mathcal{Q}}
$$


On the other hand, it is easy to see that (cf. Theorem 3.3 in [10])

$$
D_{\boldsymbol{m}, \boldsymbol{M}_{\boldsymbol{z}}}^{2}=\prod_{i=1}^{n} B_{m_{i}}^{-1}\left(z_{i}, w_{i}\right)\left(M_{z}, M_{z}^{*}\right)=\prod_{i=1}^{n} B_{m_{i}}^{-1}\left(M_{z_{i}}, M_{z_{i}}^{*}\right)=P_{\mathbb{C}}
$$

where $P_{\mathbb{C}}$ is the orthogonal projection of $A_{m}^{2}$ onto the one dimensional subspace of all constant functions. Consequently, $D_{\boldsymbol{m}, \boldsymbol{C}_{\boldsymbol{z}}}^{2}=\left.P_{\mathcal{Q}} P_{\mathbb{C}}\right|_{\mathcal{Q}}$, and hence

$$
\operatorname{rank} D_{m, C_{z}} \leq 1 \text {. }
$$

THEOREM 5.1. Let $\mathcal{Q}$ be a quotient module of $A_{m}^{2}$. Then the following conditions are equivalent:

(i) $\mathcal{Q}$ is doubly commuting.

(ii) There exists $M_{z}^{*}$-invariant closed subspace $\mathcal{Q}_{i}$ of $A_{m_{i}}^{2}, i=1, \ldots, n$, such that

$$
\mathcal{Q} \cong \mathcal{Q}_{1} \otimes \cdots \otimes \mathcal{Q}_{n}
$$

(iii) There exists Hilbert spaces $\mathcal{E}_{i}$ and partially isometric multipliers $\theta_{i} \in \mathcal{M}\left(A_{1}\left(\mathcal{E}_{i}\right), A_{m_{i}}^{2}\right)$, $i=1, \ldots, n$, such that

$$
\mathcal{Q} \cong \mathcal{Q}_{\theta_{1}} \otimes \cdots \otimes \mathcal{Q}_{\theta_{n}}
$$

where $\mathcal{Q}_{\theta_{j}}=A_{m_{j}}^{2} / \theta_{j} A_{1}\left(\mathcal{E}_{j}\right), j=1, \ldots, n$.

Proof. Let us begin by observing that the representation of $C_{z_{i}}, i=1, \ldots, n$, on $\mathcal{Q}=$ $\mathcal{Q}_{1} \otimes \cdots \otimes \mathcal{Q}_{n}$ is given by

$$
\begin{aligned}
C_{z_{i}} & =\left.P_{\mathcal{Q}}\left(I_{A_{m_{1}}^{2}} \otimes \cdots \otimes I_{A_{m_{i-1}}^{2}} \otimes M_{z} \otimes I_{A_{m_{i+1}}^{2}} \otimes \cdots \otimes I_{\mathcal{Q}_{n}}\right)\right|_{\mathcal{Q}} \\
& =\left.I_{\mathcal{Q}_{1}} \otimes \cdots \otimes I_{\mathcal{Q}_{i-1}} \otimes P_{\mathcal{Q}_{i}} M_{z}\right|_{\mathcal{Q}_{i}} \otimes I_{\mathcal{Q}_{i+1}} \otimes \cdots \otimes I_{\mathcal{Q}_{n}} .
\end{aligned}
$$

This yields $(i i) \Rightarrow(i)$ and $(i i i) \Rightarrow(i)$. The implication $(i i) \Rightarrow(i i i)$ follows from Theorem 2.3 and $(i i i) \Rightarrow(i i)$ is trivial. Hence it suffices to show $(i) \Rightarrow(i i i)$. Assume $(i)$. Then by Theorem 4.5, there exists Hilbert spaces $\mathcal{E}_{i}$ and one variable partially isometric multipliers $\Theta_{i} \in \mathcal{M}\left(A_{\hat{\boldsymbol{m}}_{i}}^{2}\left(\mathcal{E}_{i}\right), A_{\boldsymbol{m}}^{2}\left(\mathcal{D}_{\boldsymbol{m}, \boldsymbol{C}_{\boldsymbol{z}}}\right)\right), i=1, \ldots, n$, such that

$$
\left(C_{z_{1}}, \ldots, C_{z_{n}}\right) \text { on } \mathcal{Q} \cong\left(\left.P_{\mathcal{Q}_{\Theta}} M_{z_{1}}\right|_{\mathcal{Q}_{\Theta}}, \ldots,\left.P_{\mathcal{Q}_{\Theta}} M_{z_{n}}\right|_{\mathcal{Q}_{\Theta}}\right) \text { on } \mathcal{Q}_{\Theta} \text {, }
$$

and

$$
\mathcal{Q}_{\Theta}=A_{\boldsymbol{m}}^{2}\left(\mathcal{D}_{\boldsymbol{m}, \boldsymbol{C}_{\boldsymbol{z}}}\right) / \sum_{i=1}^{n} \Theta_{i} A_{\hat{\boldsymbol{m}}_{i}}^{2}\left(\mathcal{E}_{i}\right)
$$

Now by virtue of $(5.1)$ we have $\mathcal{D}_{\boldsymbol{m}, \boldsymbol{C}_{\boldsymbol{z}}} \cong\{0\}$, or $\mathbb{C}$. In order to avoid trivial considerations we assume that $\mathcal{D}_{\boldsymbol{m}, \boldsymbol{C}_{\boldsymbol{z}}} \cong \mathbb{C}$. Then

$$
\mathcal{Q} \cong \mathcal{Q}_{\Theta}=A_{\boldsymbol{m}}^{2} / \sum_{i=1}^{n} \Theta_{i} A_{\hat{\boldsymbol{m}}_{i}}^{2}\left(\mathcal{E}_{i}\right)
$$

In particular,

$$
P_{\mathcal{Q}_{\Theta}}=\prod_{i=1}^{n}\left(I_{A_{m}^{2}}-M_{\Theta_{i}} M_{\Theta_{i}}^{*}\right)=\bigotimes_{i=1}^{n}\left(I_{A_{m_{i}}^{2}}-M_{\theta_{i}} M_{\theta_{i}}^{*}\right),
$$

which implies $\mathcal{Q} \cong \mathcal{Q}_{\Theta}=\mathcal{Q}_{\theta_{1}} \otimes \cdots \otimes \mathcal{Q}_{\theta_{n}}$ and concludes the proof. 
The implication $(i) \Rightarrow(i i)$ in previous theorem was obtained in [10]. For the Hardy space case $H^{2}\left(\mathbb{D}^{n}\right)$, that is, for the case $\boldsymbol{m}=(1, \ldots, 1)$, this was observed in [9] and [14]. Moreover, as we shall see in the next section, the same result holds for more general reproducing kernel Hilbert spaces over $\mathbb{D}^{n}$.

\section{6. $\frac{1}{K}$-CALCULUS AND $K$-CONTRACTIVITY}

The key concept in our approach is the natural connections between (i) operator positivity, implemented by the inverse of a positive definite kernel function on $\mathbb{D}$, and a dilation map, again in terms of the kernel function, (ii) tensor product structure of reproducing kernel Hilbert spaces on $\mathbb{D}^{n}$, and (iii) operator positivity, implemented by the product of $n$ positive definite kernel functions on $\mathbb{D}$, of doubly commuting $n$ tuple of operators. Consequently, our considerations can be applied even for a more general framework (in the sense of Arazy and Englis [5]).

Let $k$ be a positive definite kernel function on $\mathbb{D}$ and that $k(z, w)$ is holomorphic in $z$ and anti-holomorphic in $w$, and $k(z, w) \neq 0$ for all $z, w \in \mathbb{D}$. Let $\mathcal{R}_{k} \subseteq \mathcal{O}(\mathbb{D}$, $\mathbb{C})$ be the corresponding reproducing kernel Hilbert space. Moreover, let

(i) $\mathbb{C}[z]$ is dense in $\mathcal{R}_{k}$,

(ii) the multiplication operator $M_{z}$ on $\mathcal{R}_{k}$ is a contraction,

(iii) there exists a sequence of polynomials $\left\{p_{k}\right\}_{k=0}^{\infty} \in \mathbb{C}[z, \bar{w}]$ such that

$$
p_{k}(z, \bar{w}) \longrightarrow \frac{1}{k(z, w)}, \quad(z, w \in \mathbb{D})
$$

and

$$
\sup _{k}\left\|p_{k}\left(M_{z}, M_{z}^{*}\right)\right\|<\infty .
$$

We will call such a reproducing kernel Hilbert space a standard reproducing kernel Hilbert space, or, just SRKH for short.

Let $\mathcal{R}_{k}$ be a SRKH and, by virtue of condition (i) in the above definition, let $\left\{\psi_{k}\right\}_{k=0}^{\infty} \subseteq \mathbb{C}[z]$ be an orthonormal basis of $\mathcal{R}_{k}$. For any nonnegative operator $C$ and a bounded linear operator $T$ on a Hilbert space $\mathcal{H}$, set

$$
f_{k, C}(T)=I_{\mathcal{H}}-\sum_{0 \leq m<k} \psi_{m}(T) C \psi_{m}(T)^{*} .
$$

Definition 6.1. Let $\mathcal{R}_{k}$ be a SRKH and $T \in \mathcal{B}(\mathcal{H})$. Then $T$ is said to be $k$-contractive if $\sup _{k}\left\|p_{k}\left(T, T^{*}\right)\right\|<\infty$ and

$$
C:=W O T-\lim _{k \rightarrow \infty} p_{k}\left(T, T^{*}\right)
$$

exists and nonnegative, and

$$
S O T-\lim _{k \rightarrow \infty} f_{k, C}(T)=0 .
$$

We are now ready to state the Arazy-Englis dilation result (see Corollary 3.2 in [5]).

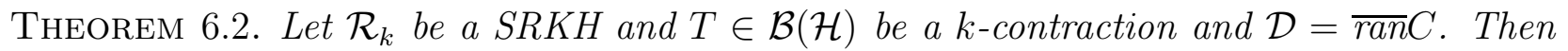
there exists an $M_{z}^{*}$-invariant closed subspace $\mathcal{Q}$ of $\mathcal{R}_{k} \otimes \mathcal{D}$ such that $\left.T \cong P_{\mathcal{Q}} M_{z}\right|_{\mathcal{Q}}$. 
In this case, the dilation map $V_{T}$ is given by (see the equality (1.5) in [5]):

$$
\left(V_{T} h\right)(z)=\sum_{k} \psi_{k}(z) \otimes C^{\frac{1}{2}} \psi_{k}(T)^{*} h . \quad(h \in \mathcal{H})
$$

Finally, note that the statement in Theorem 4.5 can be generalized in this framework as follows (see Theorem 2.3 in [15]): Let $\mathcal{H}$ be a Hilbert space and $\mathcal{S}$ be a closed subspace of $\mathcal{R}_{k} \otimes \mathcal{H}$. Then $\mathcal{S}$ is $M_{z}$-invariant if and only if $\mathcal{S}=\Theta H^{2}(\mathcal{E})$ for some Hilbert space $\mathcal{E}$ and partially isometric multiplier $\Theta \in \mathcal{M}\left(H^{2}(\mathcal{E}), \mathcal{R}_{k} \otimes \mathcal{H}\right)$.

Consequently, Theorem 2.4 holds for the class of $k$-contractions.

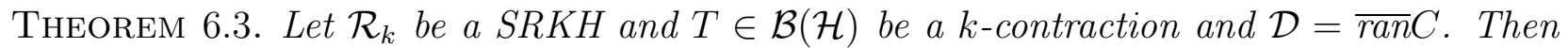
there exists a Hilbert space $\mathcal{E}$ and a partially isometric multiplier $\theta \in \mathcal{M}\left(H^{2}(\mathcal{E}), \mathcal{R}_{k} \otimes \mathcal{D}\right)$ such that $\left.T \cong P_{\mathcal{Q}_{\theta}} M_{z}\right|_{\mathcal{Q}_{\theta}}$ where $\mathcal{Q}_{\theta}=\left(\mathcal{R}_{k} \otimes \mathcal{D}\right) \ominus \theta H^{2}(\mathcal{E})$.

Now let $\mathcal{R}_{k_{i}}, i=1, \ldots, n$, be $n$ standard reproducing kernel Hilbert spaces over $\mathbb{D}$ and let

$$
\mathcal{R}_{K}:=\mathcal{R}_{k_{1}} \otimes \cdots \otimes \mathcal{R}_{k_{n}}
$$

Then $\mathcal{R}_{K}$ is a reproducing kernel Hilbert space (see Tomerlin [16]) and

$$
K(\boldsymbol{z}, \boldsymbol{w})=\prod_{i=1}^{n} k_{i}\left(z_{i}, w_{i}\right) \quad\left(\boldsymbol{z}, \boldsymbol{w} \in \mathbb{D}^{n}\right) .
$$

Let $\boldsymbol{T}$ be a doubly commuting tuple of operators on $\mathcal{H}$ and let $T_{i}$ be a $k_{i}$-contraction, $i=1, \ldots, n$. Set

$$
C_{i}=W O T-\lim _{k \rightarrow \infty} p_{i, k}\left(T_{i}, T_{i}^{*}\right),
$$

where $p_{i, k}(z, \bar{w}) \rightarrow \frac{1}{k_{i}(z, w)}, i=1, \ldots, n$. In a similar way, as in Lemma 3.1, one can prove that $C_{i} C_{j}=C_{j} C_{i}$ for all $i, j=1, \ldots, n$, and

$$
C_{\boldsymbol{T}}:=\prod_{i=1}^{n} C_{i} \geq 0
$$

By virtue of this observation, a doubly commuting tuple $\boldsymbol{T}$ is called $K$-contractive if $T_{i}$ is $k_{i}$-contractive for all $i=1, \ldots, n$ (see the remark at the end of Lemma 3.2). Consequently, all the results and proofs in this paper hold verbatim for this notion of a doubly commuting $K$-contractive tuples as well.

Acknowledgement: We are indebted to the referee for numerous comments and suggestions which improved this paper considerably. The research of the second author was supported in part by an NBHM Research Grant NBHM/R.P.64/2014.

\section{REFERENCES}

[1] J. Agler, Hypercontractions and subnormality, J. Operator Theory 13 (1985), 203-217.

[2] C. Ambrozie, M. Englis and V. Muller, Operator tuples and analytic models over general domains in $\mathbb{C}^{n}$, J. Operator Theory 47 (2002), 287-302.

[3] C. Ambrozie and D. Timotin, On an intertwining lifting theorem for certain reproducing kernel Hilbert spaces, Integral Equations Operator Theory 42 (2002), 373-384. 
[4] C. Ambrozie and D. Timotin, A von Neumann type inequality for certain domains in $\mathbb{C}^{n}$, Proc. Amer. Math. Soc. 131 (2003), 859-869.

[5] J. Arazy and M. Englis, Analytic models for commuting operator tuples on bounded symmetric domains, Trans. Amer. Math. Soc. 355 (2003), 837-864.

[6] N. Aronszajn, Theory of reproducing kernels, Trans. Amer. Math. Soc. 68 (1950), 337-404.

[7] A. Beurling, On two problems concerning linear transformations in Hilbert space, Acta Math. 81 (1949), 239-255.

[8] J. Ball and V. Bolotnikov, A Beurling type theorem in weighted Bergman spaces, C. R. Math. Acad. Sci. Paris 351 (2013), 433-436.

[9] T. Bhattacharyya, E. K. Narayanan and J. Sarkar Analytic Model of Doubly Commuting Contractions, arXiv: 1309.2384

[10] A. Chattopadhyay, B. K. Das and J. sarkar, Tensor product of quotient Hilbert modules, J. Math. Anal. Appl. 424 (2015), 727-747.

[11] R. Curto and F.-H. Vasilescu, Standard operator models in the polydisc, Indiana Univ. Math. J. 42 (1993), 791-810.

[12] V. Muller and F.-H. Vasilescu, Standard models for some commuting multioperators, Proc. Amer. Math. Soc. 117 (1993), 979-989.

[13] B. Sz.-Nagy and C. Foias, Harmonic Analysis of Operators on Hilbert Space, North Holland, Amsterdam, 1970.

[14] J. Sarkar, Jordan Blocks of $H^{2}\left(\mathbb{D}^{n}\right)$, J. Operator Theory 72 (2014) 371-385.

[15] J. Sarkar, An invariant subspace theorem and invariant subspaces of analytic reproducing kernel Hilbert spaces - I, J. Operator Theory 73 (2015), 433-441.

[16] A. Tomerlin, Products of Nevanlinna-Pick kernels and operator colligations, Integral Equations Operator Theory 38 (2000), 350-356.

Indian Institute of Science, Department of Mathematics, Bangalore, 560012, India

E-mail address: monojit12@math.iisc.ernet.in

Indian Statistical Institute, Statistics and Mathematics Unit, 8th Mile, Mysore Road, BANGALORE, 560059, INDIA

E-mail address: jay@isibang.ac.in, jaydeb@gmail.com 